\title{
Les pratiques culturales traditionnelles appauvrissent les sols en zone des savanes du Tchad
}

\author{
Michel NAITORMBAIDE ${ }^{1 *}$, François LOMPO ${ }^{2}$, Zacharie GNANKAMBARY $^{2}$, \\ Noufou OUANDAOGO ${ }^{2}$ et Michel Papaoba SEDOGO ${ }^{2}$ \\ ${ }^{1}$ Institut Tchadien de Recherche Agronomique pour le Développement (ITRAD), BP 31 Moundou, Tchad. \\ ${ }^{2}$ Institut de l'Environnement et de Recherches Agricoles (INERA), 04 BP 8645 Ouagadougou 04, \\ Burkina Faso. \\ *Auteur correspondant, E-mail : naitormbaide_michel@yahoo.fr, Tel : +23566298766/+23599129568
}

\section{RESUME}

Les systèmes de culture des savanes tchadiennes sont à base de céréales et de coton. Le mode de gestion des terres est actuellement peu adapté à la conservation de la fertilité des sols. Par conséquent, les rendements des cultures sont stationnaires. L'étude a été menée dans les savanes au sud du Tchad et a eu pour objectif d'évaluer les effets des pratiques agricoles paysannes sur les rendements des cultures. Les données ont été complétées par la caractérisation physico-chimique des sols à partir des indices de déstructuration des sols qui ont été calculés. Quatre systèmes de culture (successions culturales) ont été identifiés : coton/sorgho, arachide/sorgho, sorgho/coton et maïs/arachide. Dans ces systèmes, les rendements des cultures et l'indice de déstructuration des sols ont baissé de 2006 à 2007. De même, les teneurs en carbone des sols du système maïs/arachide ont beaucoup diminué. Les sols étudiés se sont révélés particulièrement sensibles à l'érosion et pauvres en matière organique. Pour assurer une production agricole durable, nous préconisons l'emploi d'engrais organiques et minéraux ainsi que la culture des légumineuses.

(C) 2010 International Formulae Group. All rights reserved.

Mots clés : Céréales, arachide, pratiques culturales, fertilité du sol, système de culture.

\section{INTRODUCTION}

Les systèmes de culture dans la zone des savanes du Tchad $\left(8^{\circ}-11^{\circ} \mathrm{N}\right)$ sont à base de céréales et de coton. Les pratiques culturales sont caractérisées par une utilisation très limitée des engrais minéraux et organiques qui ne compensent pas les pertes dues aux exportations des éléments minéraux par les cultures d'une part et d'autre part des résidus de cultures qui sont source de fourrage et matériaux de construction. Dans cette zone cotonnière, il apparaît que les itinéraires techniques pratiqués sont à l'origine de la dégradation de la fertilité des sols à travers leur érosion et la minéralisation accélérée de leur matière organique (Abdoulaye et al, 2006). Les résidus des cultures étant peu restitués au sol, les teneurs en matière organique baissent et par conséquent, celles de l'élément phosphore (Lompo 2008). En effet, le phosphore $(\mathrm{P})$ organique qui représente 20 à $60 \%$ du $\mathrm{P}$ total du sol est lié à la teneur et au 
degré d'évolution de la matière organique du sol (Bationo, 1998 cité par Lompo, 2008). En outre, l'extraction constante par les cultures, sans réapprovisionnement - ou avec réapprovisionnement insuffisant - d'éléments nutritifs des plantes, cause un appauvrissement constant de la fertilité du sol. Pour une agriculture attelée ou manuelle à faibles intrants et faible productivité, les restitutions et apports organiques sont un facteur majeur d'intensification (Gangry et al., 2006).

Le système d'élevage est de type traditionnel dominé par les bovins de trait et les petits ruminants. La sédentarisation des éleveurs transhumants accroît le nombre des bovins dans la zone. En effet, entre les années 1975 et 2004, le nombre des bœufs de trait de la zone est passé de 113250 à 302100 têtes (DSA, 2006). La sédentarisation des transhumants dans les zones favorables à l'élevage entraine la diminution des surfaces cultivables et la dégradation biophysique et chimique des sols.

Malgré la demande croissante en céréales d'une population qui ne cesse d'augmenter, les rendements des cultures stagnent. En effet, les rendements du sorgho par exemple en 1996 et 2005 ont été respectivement de $700 \mathrm{~kg} \mathrm{ha}^{-1}$ et $770 \mathrm{~kg} \mathrm{ha}^{-1}$ (DSA, 2006). Ces faibles rendements s'expliquent par la pauvreté naturelle des sols en éléments nutritifs et en matières organiques, l'inadaptation des systèmes de production, ainsi que par les régimes pluviométriques.

C'est dans ce contexte que les paysans ont développé plusieurs pratiques culturales dont la réduction de la durée des jachères, l'apport de fumure organique et minérale et l'association des légumineuses aux céréales. L'objectif de cette étude est d'évaluer les effets des pratiques agricoles paysannes sur les caractéristiques physico-chimiques des sols et les rendements des cultures. Nous avons émis l'hypothèse selon laquelle la non restitution des résidus de cultures et la culture continue appauvrissent les sols.

\section{MATERIEL ET METHODES Site d'étude}

L'étude a été conduite à Nguetté 1 , village situé entre $15^{\circ} 11^{\prime}$ et $15^{\circ} 15^{\prime} \mathrm{de}$ longitude Est et de $9^{\circ} 17^{\prime}$ et $9^{\circ} 24^{\prime}$ de latitude Nord (Figure 1). Le terroir couvre une superficie de 5280 ha. La pluviométrie est de type monomodal (saison hivernale d'avril à octobre) et la moyenne des dix dernières années est de $925 \mathrm{~mm}$. Les pluies sont caractérisées par une mauvaise répartition pendant la saison pluvieuse et d'une variabilité entre les années. Pendant les deux campagnes agricoles de l'étude, les pluviométries ont été caractérisées par des débuts tardifs et des arrêts brusques.

On rencontre trois types de sol à Nguetté 1 :

- des îlots de lithosols, peu profonds et parsemant l'Est et l'Ouest du terroir utilisés comme aires de pâturage. Ces sols sont aptes à la culture du sorgho (Sorghum bicolor), du sésame (Sesamum indicum), du niébé (Vigna unguiculata) et de l'arachide (Arachis hypogaea) ;

- les sols colluviaux où sont implantés les vergers;

- et les sols ferralitiques où sont cultivées le sorgho, le coton (Gossypium hirsutum) et l'arachide.

\section{Matériel}

Le matériel végétal est composé des quatre principales espèces végétales les plus cultivées dans le village. Il s'agit du sorgho (variété local rouge), de l'arachide (57 - 313), du coton (A51) et du maïs (Zea mays), variété CMS 8701.

Les fumures orgnaniques et minérales sont celles utilisées par les producteurs. Il s'agit du fumier de parc et des engrais 
minéraux (NPKSB 19-12-19-5-1,2 ; urée 46\% $\mathrm{N})$ utilisés pour la production du coton.

\section{Méthodes \\ Méthodes d'enquête et d'évaluation des rendements des cultures}

Les analyses des pratiques ont exploré les systèmes de culture et leurs performances sur 2 années (2006 et 2007) auprès de 10 producteurs. Pour choisir les exploitants, un échantillonnage systématique a été opéré à partir de la liste d'une association villageoise de Nguetté 1 qui compte 120 adhérents. Sur cette liste, à partir d'un numéro d'ordre choisi aléatoirement, on a retenu le $9^{\mathrm{e}}$ exploitants. Les suivis agronomiques ont concerné 30 stations (surface homogène du point de vue terrain et système de culture) dans les champs paysans.

Au cours des années 2006 et 2007, quatre types de successions culturales ont été suivis : coton/sorgho; sorgho/coton ; arachide/sorgho ; maïs/arachide. Celles-ci seront assimilées aux systèmes de culture dans la suite des analyses.

Les superficies des stations (placettes) ont été de $72 \mathrm{~m}^{2}\left(24 \mathrm{~m}^{2} \times 3\right)$ pour le coton, le maïs et le sorgho et de $75 \mathrm{~m}^{2}\left(25 \mathrm{~m}^{2} \times 3\right)$ pour l'arachide. Les différentes mesures et observations ont été effectuées dans les stations implantées en 3 répétitions par champ paysan.

\section{Méthodes d'analyse des sols}

L'évaluation de la fertilité physique et chimique des sols a porté sur 60 échantillons composites de sols prélevés dans la profondeur de $0-20 \mathrm{~cm}$. Les analyses ont été effectuées dans les laboratoires de Saria et de Kamboinsé de l'Institut de l'Environnement et de Recherches Agricoles au Burkina Faso. Pour déterminer les teneurs en azote et le phosphore total, les échantillons de sols ont été d'abord minéralisés à l'aide d'un mélange $\mathrm{H}_{2} \mathrm{SO}_{4}-\mathrm{Se}-\mathrm{H}_{2} \mathrm{O}_{2}$ à $450{ }^{\circ} \mathrm{C}$ pendant $4 \mathrm{~h}$ (Bremner 1965). Puis, les teneurs en $\mathrm{P}$ et $\mathrm{N}$ dans les minéralisats ont été déterminées à l'aide d'un colorimètre automatique (Skalar SANplus Segmented flow analyzer, Model 4000-02, Breda, Holland). Les teneurs en K échangeable ont été dosées au spectrophotomètre à émission de flammes. La méthode de Bray-2 a été utilisée pour la détermination du phosphore assimilable. Le carbone a été déterminé par la méthode de Walkley et Black (1934). La granulométrie a été déterminée par la méthode de la pipette Robinson. Elle a permis de calculer l'indice de déstructuration du sol, $\mathrm{St}(\%)=\mathrm{M} . \mathrm{O} /$ $(\mathrm{A}+\mathrm{L}) \times 100$ (Emerson, 1967 et Jones, 1976, cités par Pieri, 1989).

Le $\mathrm{pH}$ a été mesuré par la méthode électrométrique avec des rapports sol/solution de $1 / 2,5$.

La méthode utilisée pour le fractionnement granulométrique de la matière organique est inspirée de celle élaborée par Feller (1979). Elle a consisté en la séparation de trois fractions comportant chacune une partie minérale (PM) et une partie organique (PO). La $1^{\text {re }}$ fraction rassemble les particules ayant une taille supérieure à $200 \mu \mathrm{m}$ (sables grossiers). Celles de la $2^{\mathrm{e}}$ fraction rassemble la fraction comprise entre $50 \mu \mathrm{m}$ et $200 \mu \mathrm{m}$ (sables fins). La $3^{\mathrm{e}}$ fraction regroupe les éléments inférieurs à $50 \mu \mathrm{m}$ (fraction argilolimoneuse).

\section{Traitements statistiques}

Les données collectées ont été soumises à une analyse de variance (ANOVA) à l'aide de SPSS 10.1. Les différences sont significatives lorsque $\mathrm{p}<0,05$ suivant le test de Duncan.

\section{RESULTATS}

Systèmes de culture

Principales espèces cultivées et répartition spatiale

La culture de sorgho occupe $33 \%$ de la superficie totale cultivée alors que la culture de maïs n'occupe que $13 \%$ de celle-ci (Tableau 1).

Toutes les parcelles suivies sont cultivées depuis au moins 5 ans. Les plus anciennes sont celles qui portent le maïs, proches des cases.

\section{Successions et associations culturales}

Chez les exploitants échantillonnés, quatre principales successions culturales ont 
été identifiés : coton/sorgho; sorgho/coton ; arachide/sorgho ; maïs/arachide.

Le coton est en tête d'une succession culturale dans $26 \%$ des cas recensés. Lorsque la jachère est de courte durée (2 à 3 ans), ce sont le sorgho ( $16 \%$ des cas), le maïs $(7 \%)$ et l'arachide (7\%) qui viennent en tête des successions culturales. Lorsque le niébé est intégré dans le système de production, cette culture vient également en tête des successions culturales ( $40 \%$ des cas).

L'étude a révélé cinq types d'associations culturales: mil + arachide, sorgho + arachide, niébé + sorgho, mil + niébé et maïs + niébé.

Gestion de la fertilité des sols et les rendements des cultures

La fumure organique est très peu appliquée sur les cultures. Seulement $50 \%$ des exploitants apportent du fumier de parc sur le sorgho et le maïs. Les doses appliquées sont très faibles (100 à $280 \mathrm{~kg} \mathrm{ha}^{-1}$ ).

Dans les parcelles suivies en 2006, ce sont seulement les cultures de coton et de maïs qui ont reçu les engrais minéraux. Le coton a reçu $75 \mathrm{~kg} \mathrm{ha}^{-1}$ de NPKSB et $50 \mathrm{~kg}$ $\mathrm{ha}^{-1}$ d'urée. Le maïs n'a reçu que $50 \mathrm{~kg} \mathrm{ha}^{-1}$ de NPKSB et $25 \mathrm{~kg} \mathrm{ha}^{-1}$ d'urée. En 2007, seul le coton a reçu en moyenne $109 \mathrm{~kg} \mathrm{ha}^{-1} \mathrm{de}$ NPKSB et $30 \mathrm{~kg} \mathrm{ha}{ }^{-1}$ d'urée. Cette augmentation de la dose de l'engrais minéral NPKSB n'a pas induit une augmentation de rendement en coton grain (Tableau 2). Le test de Duncan au seuil de 5\% n'est significatif que pour les rendements de l'arachide pendant les deux années de suivi.

Evolution des caractéristiques physicochimiques des sols

Evolution des caractéristiques physiques des sols

Les sols du site de l'étude sont à texture sablo-limoneuse. Les successions culturales pratiquées ont augmenté les teneurs des sols en argile (Tableau 3). Le test de Duncan est significatif au seuil de $5 \%$ pour les teneurs moyennes en argile entre la $1^{\text {re }}$ et la $2^{\mathrm{e}}$ année dans la succession culturale sorgho/coton.
L'indice de déstructuration (St \%) des sols dans les systèmes de culture étudiés a baissé d'une à quatre unités, rendant ainsi les sols très sensibles à l'érosion à la $2^{\mathrm{e}}$ année de suivi.

- Le carbone et l'azote total des sols

A la $1^{\text {re }}$ année de l'étude (2006), les sols du système de culture maïs/arachide ont les plus fortes teneurs en $\mathrm{C}$-total et $\mathrm{N}$-total. Dans les systèmes de culture coton/sorgho, arachide/sorgho et sorgho/coton, les teneurs en ces éléments sont similaires (Tableau 4).

A la $2^{\mathrm{e}}$ année de culture, les sols des systèmes arachide/sorgho, sorgho/coton et maïs/arachide se sont relativement appauvris en matière organique par rapport à la $1^{\text {re }}$ année de culture. Cependant, le test de Duncan au seuil de $5 \%$ n'a pas révélé de différence significative en cet élément à la $2^{\mathrm{e}}$ année de culture dans les sols de tous les systèmes de culture étudiés.

Pendant la $1^{\text {re }}$ année de suivi, le rapport $\mathrm{C} / \mathrm{N}$ des sols de tous les systèmes sont élevés. A la $2^{\mathrm{e}}$ année, ces rapports ont baissé significativment pour les systèmes arachide/sorgho et maïs/arachide.

\section{Evolution des caractéristiques chimiques des} sols

Les $\mathrm{pH}$ des systèmes de culture étudiés ont varié de 6,9 à 7,1 à la $1^{\text {re }}$ année. Le test Duncan seuil de $5 \%$ n'a révélé aucune différence significative entre eux. A la $2^{\mathrm{e}}$ année, les pH ont varié de 6,1 à 6,3 . Le test Duncan seuil de $5 \%$ a révélé de différence significative entre les $\mathrm{pH}$ des systèmes de culture étudiés. Les $\mathrm{pH}$ des systèmes archide/sorgho, sorgho/coton et maïs/arachide ont relativement baissé (Tableau 5).

Les teneurs en phosphore total ont diminué significativment dans le système maïs/arachide. Pour les autres systèmes, la tendance générale en cet élément est en baisse mais pas significative.

Les teneurs en potassium dans les systèmes arachide/sorgho, sorgho/coton et maïs/arachide ont connu des baisses respectives de $-26 ;-20$ et $-20 \mathrm{mg} \mathrm{kg}^{-1}$ entre la $1^{\text {re }}$ et la $2^{\mathrm{e}}$ année de suivi. 


\section{Fraction granulométrique de la matière organique du sol}

Les teneurs en carbone dans les quatre systèmes de culture sont plus élevées dans la fraction argilo-limoneuse $\mathrm{F}(0-50 \quad \mu \mathrm{m})$, comparativement aux autres fractions (Tableau 6). Les teneurs en carbone ont varié entre 3,20 et $6,0 \mathrm{~g} \mathrm{~kg}^{-1}$ sol dans la fraction $\mathrm{F}$ $(0-50 \mu \mathrm{m})$ et entre 0,22 et $0,67 \mathrm{~g} \mathrm{~kg}^{-1}$ sol dans la fraction $\mathrm{F}(50-200 \mu \mathrm{m})$ et entre 0,14 et $0,61 \mathrm{~g} \mathrm{~kg}^{-1}$ sol dans la fraction $(200-2000$ $\mu \mathrm{m})$. Ces teneurs, évaluées en termes de proportions par rapport au C-total, ont varié entre 82,42 à $89,89 \%$ dans la fraction argilolimoneuse, entre 9,18 et $9,89 \%$ dans la fraction de sable fin et entre 3,93 et $8,38 \%$ de sable grossier.

La teneur en carbone de la fraction argilo-limoneuse $(0-50 \mu \mathrm{m})$ et de sables fins $(50-200 \mu \mathrm{m})$ des sols du système de culture maï/arachide est significativement plus élevé que celle des autres systèmes. Dans la fraction de sables grossiers (200 -2 $000 \mu \mathrm{m})$, les teneurs du carbone des 4 systèmes étudiés ne sont pas significativement différentes selon le test de Duncan au seuil de 5\%.

Les teneurs des sols en azote des sols des systèmes de culture suivis ont varié entre 0,02 et $0,42 \mathrm{~g} \mathrm{~kg}^{-1}$ sol (Tableau 6). La fraction argilo-limoneuse $(0-50 \mu \mathrm{m})$ contient entre 0,27 et $0,42 \mathrm{~g} \mathrm{~kg}^{-1}$ sol. Dans la fraction du sable fin $(50-200 \mu \mathrm{m})$, les teneurs en azote des sols sont relativement faibles. Elles n'ont varié qu'entre 0,03 et $0,04 \mathrm{~g} \mathrm{~kg}^{-1}$ sol. Dans la fraction de sables grossiers des sols (200 $2000 \mu \mathrm{m}$ ), l'azote est quasiment inexistant car ses teneurs n'ont varié qu'entre 0,02 et $0,04 \mathrm{~g} \mathrm{~kg}^{-1}$ sol. Dans les 3 fractions des sols étudiés, le test de Duncan n'a révélé aucune différence au seuil de 5\% entre les teneurs en azote dans les systèmes de culture étudiés.

Le rapports $\mathrm{C} / \mathrm{N}$ de la matière organique associée aux particules argilolimoneuses $\left(\begin{array}{lll}0 & -50 & \mu \mathrm{m}\end{array}\right)$ dans le système coton/sorgho est de 11,6; dans le système arachide/sorgho, il est de 14; dans les systèmes sorgho/coton et maïs/arachide, il est respectivement de 12,7 et 15 .

Dans la fraction $(50-200 \mu \mathrm{m})$, le rapport $\mathrm{C} / \mathrm{N}$ est de 8,$3 ; 18,1 ; 13,5$ et 17,2 respectivement dans les systèmes de culture coton/sorgho, arachide/sorgho, sorgho/coton et maïs/arachide. Par contre, dans la fraction $(200-2000 \mu \mathrm{m})$, le rapports $\mathrm{C} / \mathrm{N}$ est de 6,1 dans coton/sorgho; 13 dans le système arachide/sorgho ; de 8,5 et 16,2 respectivement dans les systèmes sorgho/coton et maïs/arachide. Globalement, les rapports $\mathrm{C} / \mathrm{N}$ augmentent de la fraction fine à la fraction sableuse dans le système maïs/arachide tandis que l'évolution inverse est observée dans les systèmes coton/sorgho, arachide/sorgho et sorgho/coton.

Tableau 1 : Caractéristiques des parcelles étudiées (superficies moyennes cultivées par espèce dans chaque exploitation et leurs importances relatives dans l'assolement, les distances qui séparent les parcelles cultivées des exploitations et les durées de leurs mises en culture).

\begin{tabular}{lcccc}
\hline Culture & $\begin{array}{c}\text { Superficie } \\
\text { moyenne } \\
\text { (ha) }\end{array}$ & $\begin{array}{c}\text { Proportion de la } \\
\text { superficie totale des } \\
\text { cultures } \\
(\boldsymbol{\%})\end{array}$ & $\begin{array}{c}\text { Distance exploitation- } \\
\text { parcelle } \\
(\mathbf{k m})\end{array}$ & $\begin{array}{c}\text { Age de mise en } \\
\text { valeur } \\
(\text { ans })\end{array}$ \\
\hline Arachide & 1,3 & 24,1 & {$[1-2]$} & {$[5-20]$} \\
Coton & 1,3 & 24,1 & {$[1-1,5]$} & {$[7-22]$} \\
Maïs & 0,7 & 13,0 & {$[0,5-1]$} & {$[21-28]$} \\
Sorgho & 1,8 & 33,3 & {$[3-7]$} & {$[7-9]$} \\
Autres & 0,3 & 5,5 & - & - \\
\hline
\end{tabular}


Tableau 2 : Rendements $\left(\mathrm{kg} \mathrm{ha}^{-1}\right)$ des spéculations mentionnées entre parenthèses dans les successions culturales.

\begin{tabular}{lcccc}
\hline Années & $\begin{array}{c}\text { Coton/sorgho } \\
\text { et sorgho/coton } \\
\text { (Coton) }\end{array}$ & $\begin{array}{c}\text { Arachide/sorgho } \\
\text { et maïs/arachide } \\
\text { (Arachide) }\end{array}$ & $\begin{array}{c}\text { Sorgho/coton } \\
\text { et coton/sorgho } \\
\text { (Sorgho) }\end{array}$ & $\begin{array}{c}\text { Maïs/arachide } \\
\text { (Maïs) }\end{array}$ \\
\hline 2006 & $896 \pm 88 \mathrm{a}$ & $2800 \pm 200 \mathrm{a}$ & $1458 \pm 493 \mathrm{a}$ & $2206 \pm 746$ \\
2007 & $909 \pm 147 \mathrm{a}$ & $1267 \pm 94 \mathrm{~b}$ & $1173 \pm 145 \mathrm{a}$ & \\
\hline
\end{tabular}

Dans une même colonne, les valeurs suivies de la même lettre ne sont pas significativement différentes au seuil de 5\% selon le test de Duncan $(n=10)$.

Tableau 3 : Teneur en argile, limon et sable des stations d'observation en 2006 et 2007.

\begin{tabular}{lcccccccc}
\hline \multirow{2}{*}{$\begin{array}{l}\text { Succession } \\
\text { culturale }\end{array}$} & \multicolumn{2}{c}{ Argile (\%) } & \multicolumn{2}{c}{ Limon (\%) } & \multicolumn{2}{c}{ Sable (\%) } & \multicolumn{2}{c}{ St (\%) } \\
\cline { 2 - 9 } & $\mathbf{2 0 0 6}$ & $\mathbf{2 0 0 7}$ & $\mathbf{2 0 0 6}$ & $\mathbf{2 0 0 7}$ & $\mathbf{2 0 0 6}$ & $\mathbf{2 0 0 7}$ & $\mathbf{2 0 0 6}$ & $\mathbf{2 0 0 7}$ \\
\hline Coton/sorgho & $6,3 \mathrm{a}$ & $7,4 \mathrm{a}$ & $12,4 \mathrm{a}$ & $14,2 \mathrm{a}$ & $81,3 \mathrm{a}$ & $78,4 \mathrm{a}$ & $5 \mathrm{a}$ & $4 \mathrm{a}$ \\
Arachide/sorgho & $6,3 \mathrm{a}$ & $7,4 \mathrm{a}$ & $12,4 \mathrm{a}$ & $14,2 \mathrm{a}$ & $81,3 \mathrm{a}$ & $78,4 \mathrm{a}$ & $5 \mathrm{a}$ & $4 \mathrm{a}$ \\
Sorgho/coton & $5,0 \mathrm{~b}$ & $8,2 \mathrm{a}$ & $12,6 \mathrm{a}$ & $15,6 \mathrm{a}$ & $82,5 \mathrm{a}$ & $76,3 \mathrm{a}$ & $5 \mathrm{a}$ & $3 \mathrm{a}$ \\
Maïs/arachide & $5,6 \mathrm{a}$ & $9,0 \mathrm{a}$ & $14,8 \mathrm{a}$ & $9,4 \mathrm{a}$ & $79,6 \mathrm{a}$ & $81,6 \mathrm{a}$ & $6,5 \mathrm{a}$ & $2,5 \mathrm{a}$ \\
\hline
\end{tabular}

Pour une même caractéristique, les valeurs suivies de la même lettre dans une même colonne ne sont pas significativement différentes au seuil de 5\% selon le test de Duncan.

Tableau 4 : Effet des successions culturales sur les teneurs des sols en carbone total $\left(\mathrm{g} \mathrm{kg}^{-1}\right)$ et en azote total $\left(\mathrm{g} \mathrm{kg}^{-1}\right)$.

\begin{tabular}{lcccccc}
\hline \multirow{2}{*}{$\begin{array}{l}\text { Succession } \\
\text { culturale }\end{array}$} & \multicolumn{2}{c}{ Carbone } & \multicolumn{2}{c}{ Azote } & \multicolumn{2}{c}{ C/N } \\
\cline { 2 - 7 } & $\mathbf{2 0 0 6}$ & $\mathbf{2 0 0 7}$ & $\mathbf{2 0 0 6}$ & $\mathbf{2 0 0 7}$ & $\mathbf{2 0 0 6}$ & $\mathbf{2 0 0 7}$ \\
\hline Coton/sorgho & $4,4 \pm 0,4 \mathrm{a}$ & $4,5 \pm 1,1 \mathrm{a}$ & $0,3 \pm 0,1 \mathrm{a}$ & $0,4 \pm 0,1 \mathrm{a}$ & $15 \pm 2,4 \mathrm{a}$ & $10 \pm 2,0 \mathrm{a}$ \\
Arachide/sorgho & $5,5 \pm 1,1 \mathrm{a}$ & $4,4 \pm 0,4 \mathrm{a}$ & $0,4 \pm 0,1 \mathrm{a}$ & $0,5 \pm 0 \mathrm{a}$ & $14 \pm 0,4 \mathrm{a}$ & $9 \pm 1,3 \mathrm{~b}$ \\
Sorgho/coton & $5,5 \pm 0,9 \mathrm{a}$ & $3,8 \pm 1,9 \mathrm{a}$ & $0,4 \pm 0,1 \mathrm{a}$ & $0,3 \pm 0,1 \mathrm{a}$ & $13 \pm 1,7 \mathrm{a}$ & $10 \pm 3,5 \mathrm{a}$ \\
Maïs/arachide & $7,9 \pm 0,5 \mathrm{a}$ & $3,0 \pm 0,1 \mathrm{~b}$ & $0,6 \pm 0 \mathrm{a}$ & $0,4 \pm 0,1 \mathrm{a}$ & $13 \pm 0,5 \mathrm{a}$ & $7 \pm 1,3 \mathrm{~b}$ \\
\hline
\end{tabular}

Pour une même caractéristique, les valeurs suivies de la même lettre dans une même colonne ne sont pas significativement différentes au seuil de $5 \%$ selon le test de Duncan $(n=30)$.

Tableau 5 : Effet à court terme des successions culturales sur le $\mathrm{pH}$ du sol, et les teneurs des sols en phosphore $\left(\mathrm{mg} \mathrm{kg}^{-1}\right)$ et potassium $\left(\mathrm{mg} \mathrm{kg}^{-1}\right)$.

\begin{tabular}{lcccccc}
\hline \multirow{2}{*}{$\begin{array}{l}\text { Successions } \\
\text { culturales }\end{array}$} & \multicolumn{6}{c}{ Caractéristiques physico - chimiques } \\
\cline { 2 - 7 } & \multicolumn{2}{c}{ pH eau } & \multicolumn{2}{c}{ Phosphore Total } & \multicolumn{2}{c}{ Potassium total } \\
\cline { 2 - 7 } & 2006 & 2007 & 2006 & 2007 & 2006 & 2007 \\
\hline Coton/sorgho & $6,9 \pm 0 \mathrm{a}$ & $6,2 \pm 0,2 \mathrm{a}$ & $84,9 \pm 19,7 \mathrm{a}$ & $94,6 \pm 11,7 \mathrm{a}$ & $194 \pm 67 \mathrm{a}$ & $205 \pm 56 \mathrm{a}$ \\
Arachide/sorgho & $7,0 \pm 0,3 \mathrm{a}$ & $6,1 \pm 0,5 \mathrm{~b}$ & $117,2 \pm 28,9 \mathrm{a}$ & $94,6 \pm 8,3 \mathrm{a}$ & $304 \pm 39 \mathrm{a}$ & $278 \pm 87 \mathrm{a}$ \\
sorgho/coton & $7,0 \pm 0,2 \mathrm{a}$ & $6,1 \pm 0,2 \mathrm{~b}$ & $87,1 \pm 17,5 \mathrm{a}$ & $94,6 \pm 16,5 \mathrm{a}$ & $246 \pm 81 \mathrm{a}$ & $226 \pm 61 \mathrm{a}$ \\
maïs/arachide & $7,1 \pm 0,1 \mathrm{a}$ & $6,3 \pm 0,1 \mathrm{~b}$ & $140,4 \pm 0,3 \mathrm{a}$ & $102,9 \pm 11,7 \mathrm{~b}$ & $309 \pm 39 \mathrm{a}$ & $289 \pm 43 \mathrm{a}$ \\
\hline
\end{tabular}

Pour une même caractéristique, les valeurs suivies de la même lettre dans une même colonne ne sont pas significativement différentes au seuil de $5 \%$ selon le test de Duncan, $(n=30)$. 
Tableau 6 : Teneurs en carbone total et en azote total $\left(\mathrm{g} \mathrm{kg}^{-1}\right)$ des fractions granulométriques.

\begin{tabular}{|c|c|c|c|c|c|c|}
\hline \multirow[t]{2}{*}{$\begin{array}{l}\text { Système de } \\
\text { culture }\end{array}$} & \multicolumn{3}{|c|}{$\begin{array}{l}\text { Teneur en carbone total }\left(\mathrm{g} \mathrm{kg}^{-1}\right) \\
\text { dans les } 3 \text { fractions }(\mu \mathrm{m})\end{array}$} & \multicolumn{3}{|c|}{$\begin{array}{c}\text { Teneur en azote total }\left(\mathrm{g} \mathrm{kg}^{-1}\right) \text { dans les } \\
\mathbf{3} \text { fractions }(\mu \mathrm{m})\end{array}$} \\
\hline & $0-50$ & $50-200$ & $200-2000$ & $0-50$ & $50-200$ & $200-2000$ \\
\hline Coton/sorgho & $3,20 \pm 0,5 b$ & $0,22 \pm 0,1 \mathrm{~b}$ & $0,14 \pm 0 \mathrm{a}$ & $0,27 \pm 0 \mathrm{a}$ & $0,03 \pm 0 \mathrm{a}$ & $0,02 \pm 0 \mathrm{a}$ \\
\hline Arachide/sorgho & $4,28 \pm 1,0 \mathrm{~b}$ & $0,48 \pm 0,2 \mathrm{ab}$ & $0,41 \pm 0,3 \mathrm{a}$ & $0,30 \pm 0 \mathrm{a}$ & $0,03 \pm 0 \mathrm{a}$ & $0,03 \pm 0 \mathrm{a}$ \\
\hline Sorgho/coton & $3,94 \pm 0,5 b$ & $0,47 \pm 0 \mathrm{ab}$ & $0,34 \pm 0 \mathrm{a}$ & $0,31 \pm 0 \mathrm{a}$ & $0,04 \pm 0 \mathrm{a}$ & $0,04 \pm 0 \mathrm{a}$ \\
\hline Maïs/arachide & $6,00 \pm 0,5 \mathrm{a}$ & $0,67 \pm 0 \mathrm{a}$ & $0,61 \pm 0 \mathrm{a}$ & $0,42 \pm 0 \mathrm{a}$ & $0,04 \pm 0 \mathrm{a}$ & $0,04 \pm 0 \mathrm{a}$ \\
\hline
\end{tabular}

Dans une même colonne, les valeurs suivies de la même lettre ne sont pas significativement différentes au seuil de 5\% selon le test de Duncan, $(\mathrm{n}=30)$.

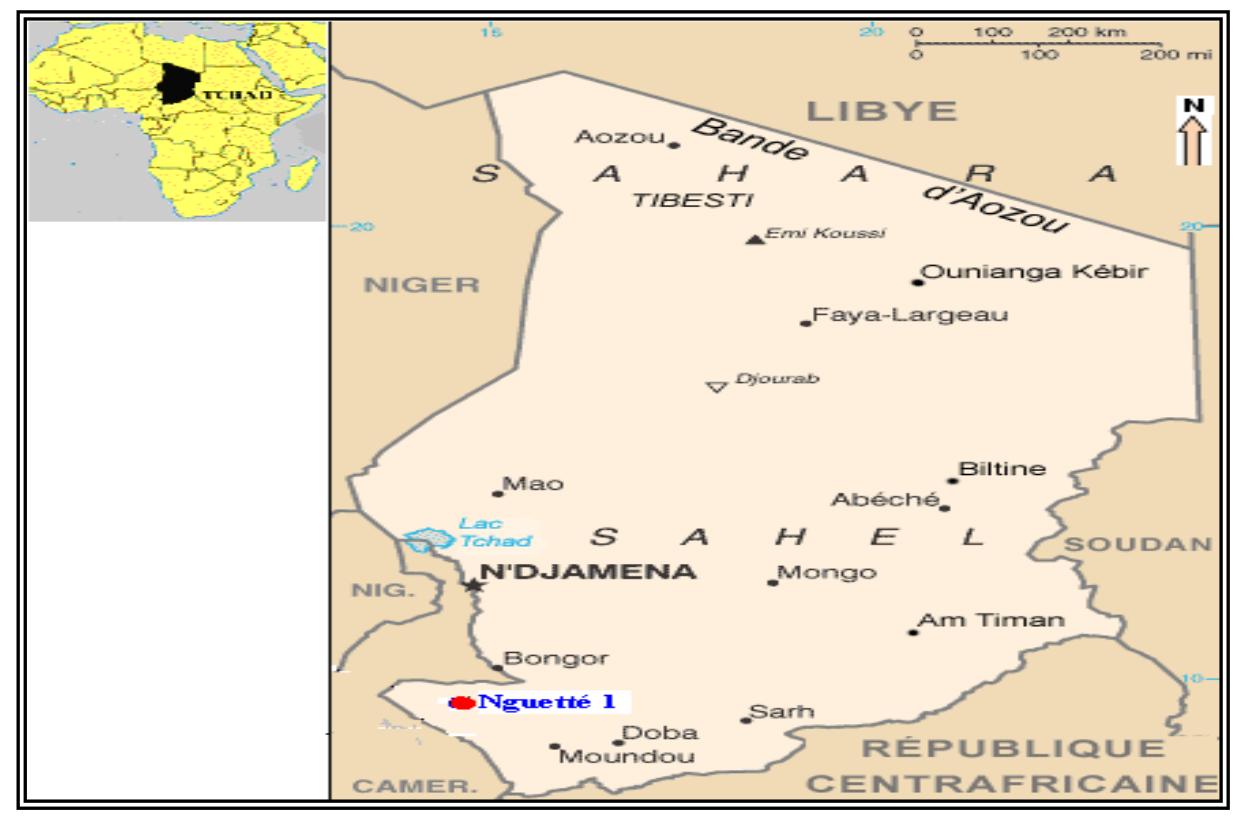

Figure 1: Localisation du site d'étude.

\section{DISCUSSION}

Systèmes de culture

Espèces cultivées et répartition spatiale

Les cultures sont implantées en cercles concentriques autour des exploitations agricoles dans l'ordre suivant: le maï; le coton; l'arachide et le sorgho. Selon les exploitants, l'emplacement d'une culture « est lié à la force de la terre », c'est-à-dire le niveau de la fertilité de celle-ci. Le maïs cultivé autour des cases est donc sur les terres les plus fertiles.

Le coton et l'arachide ont été cultivés sur des superficies relativement importantes. En effet, compte tenu de la crise de la filière cotonnière et du débouché de vente vers le Cameroun, les paysans adoptent d'autres stratégies pour accroître leurs revenus. L'arachide est de ce point de vue en passe de devenir tout comme le coton, une importante 
culture de rente. Djondang (2003) a aussi montré que les proportions des producteurs d'arachide varient de 30 à $90 \%$ dans les savanes du Tchad. Bien que l'arachide enrichit le sol en azote de 8 à $23 \mathrm{~kg}-\mathrm{N}$ ha ${ }^{-1}$ par la fixation de l'azote atmosphérique (Bado, 2002) et contribue dans une certaine mesure à améliorer les revenus des producteurs, la pratique culturale actuelle peut être à terme un facteur de paupérisation du capital sol. En effet, les exportations minérales ne sont que faiblement compensées, tandis que les durées des jachères ne sont plus suffisamment longues pour permettre l'amélioration de la fertilité du sol.

\section{Successions et associations culturales}

La succession culturale est une pratique répandue dans les exploitations agricoles échantillonnées. Elles permettraient aux exploitants de diversifier leurs revenus et d'améliorer la fertilité de leurs sols.

L'association culturale est pratiquée dans toutes les exploitations échantillonnées. L'étude de Nuttens (2001) menée dans la zone des savanes tchadiennes a également montré que cette pratique concerne entre 65 à $85 \%$ d'exploitants agricoles. L'association des légumineuses et des céréales présente plusieurs avantages qui sont entre autres : la fixation de l'azote atmosphérique par la légumineuse, la maîtrise des adventices, l'amélioration de teneur du sol en matière organique et en éléments minéraux. En outre, l'association des cultures peut aussi permettre de limiter les risques bioclimatiques afin d'assurer un minimum de production.

\section{Gestion de la fertilité des sols et les} rendements des cultures

Les apports organiques sont quasiment négligés par la plupart des producteurs. Or, elle est une alternative pour l'amélioration ou le maintien de la fertilité des sols eu égard aux prix des engrais et leur indisponibilité. Les exploitations regorgent de potentialités, mais le faible niveau d'équipement ne permet pas aux producteurs de produire le fumier ou le compost pour maintenir ou améliorer la fertilité des sols. Les doses appliquées aux cultures qui varient entre 100 à $280 \mathrm{~kg} \mathrm{ha}^{-1}$ et ne permettent pas d'assurer la durabilité des systèmes de culture identifiés actuels. En effet, pour assurer une production durable, la dose minimale de fumier à apporter tous les 2 à 3 ans est de $5 \mathrm{t} \mathrm{ha}^{-1}$ (Berger, 1996).

En dehors de la Cotontchad qui fournit aux cotonculteurs à crédit les engrais minéraux, la filière engrais est quasiment inexistante au Tchad. Ceci rend pratiquement impossible l'application d'engrais minéraux sur les céréales.

Les engrais minéraux sont souvent appliqués aux cultures sans le fumier. Cette pratique entraîne l'acidification des sols dans la mesure où l'engrais n'est pas associé à la fumure organique (Sedogo, 1993).

Ces pratiques ont toutefois permis aux exploitants d'obtenir des rendements bien meilleurs que ceux obtenus dans la région pendant les mêmes campagnes agricoles. En effet, pendant l'année 2007, les rendements des cultures sont de $746 \mathrm{~kg} \mathrm{ha}^{-1}$ pour le coton, $965 \mathrm{~kg} \mathrm{ha}^{-1}$ pour l'arachide, $760 \mathrm{~kg} \mathrm{ha}^{-1}$ pour le sorgho et de $834 \mathrm{~kg} \mathrm{ha}^{-1}$ pour le maïs. Cela peut s'expliquer par le dispositif de suivi mis en place et qui a permis de mieux collecter les données car réduit à de petits espaces. Toutefois, une baisse relative des rendements des cultures suivies a été observée. Celle-ci n'est pas uniquement due aux pratiques agricoles paysannes peu adaptées à la conservation de la fertilité des sols, mais aussi, à l'arrêt précoce des pluies.

\section{Evolution des caractéristiques physico- chimiques des sols}

Les sols des systèmes de culture étudiés ont un indice de déstructuration (St) inférieur à 9\%. Selon Emerson (1967) et Jones (1976), cités par Pieri (1989), si St<5\%, le sol est très sensible à l'érosion; si $\mathrm{St}<7 \%$, le sol est moyennement sensible à l'érosion, si $\mathrm{St}>9 \%$, le sol n'est pas sensible à l'érosion. A la $1^{\text {re }}$ année de suivi, seule la parcelle du système de culture coton/sorgho a été très sensible à 
l'érosion. Par contre les parcelles des autres systèmes de culture ont été moyennement sensibles. A la $2^{\mathrm{e}}$ année, toutes les parcelles des systèmes de culture étudiés ont été très sensibles à l'érosion.

A la $2^{\mathrm{e}}$ année, les teneurs des sols en matière organique et les rapports $\mathrm{C} / \mathrm{N}$ ont suivi la même tendance dans tous les systèmes de culture. Pendant cette année, les teneurs en carbone ont aussi relativement baissé dans certains systèmes. Elles n'ont varié qu'entre 3 et $4,5 \mathrm{~g} \mathrm{~kg}^{-1}$, soit 5,2 et $7,8 \mathrm{~g} \mathrm{~kg}^{-1}$ de matière organique. Ces résultats montrent que les teneurs en matière organique des sols des savanes du Tchad connaissent une évolution régressive. En effet, Richard et Djoulet (1986) ont déjà montré que dans cette zone, les teneurs en matière organique variaient entre 5,9 et $8,5 \mathrm{~g} \mathrm{~kg}^{-1}$. La mise en culture des parcelles dans tous les systèmes de culture étudiés se fait sans apport organique conséquent. Cette pratique a diminué les teneurs en matière organique des parcelles suivies. Balesdent et al. (2000) ont aussi observé que une telle pratique entraîne une baisse annuelle de -2 à $-4 \%$ par an selon les situations agro-écologiques.

La variation des indices de déstructuration des teneurs en carbone sont particulièrement importantes dans le système de culture maïs/arachide. En effet, malgré l'apport du fumier à la $1^{\text {re }}$ année, les sols se sont appauvris en matière organique. Cela peut s'expliquer par le fait que les résidus de l'arachide et du maïs cultivés on été totalement exportés par les exploitants comme aliment de bétail ou les animaux transhumants. En plus, les résidus de ces cultures se décomposent rapidement. Sedogo (1993) a également observé que cette pratique culturale entraîne une perte de la fertilité des sols.

Les sols dans les successions culturales étudiés sont pauvres en phosphore et en potassium totaux. Les faibles teneurs en ces éléments seraient liées aux mauvaises pratiques culturales telles que les brûlis des résidus de récolte, le faible apport d'engrais organiques et minéraux, les exportations des résidus des récoltes sans une fertilisation potassique conséquente, mais aussi à l'érosion hydrique. Pieri (1989) a également rapporté que les résidus exportés peuvent mobiliser $100 \mathrm{~kg} \mathrm{ha}^{-1}$ de $\mathrm{K}_{2} 0$ dans les conditions favorables de culture.

Le fractionnement granulométrique a montré que la matière organique des sols des systèmes de culture étudiés se retrouve principalement dans la fraction liée aux particules argilo-limoneuses $(<50 \mu \mathrm{m})$. Ces résultats corroborent ceux de Christensen (2001) en zone tempérée. Les mêmes observations ont été faites par Pallo et al. (2009) au Burkina Faso.

Les quantités de carbone liées à la fraction argilo -limoneuse est particulièrement importante dans les sols des systèmes de culture coton/sorgho et sorgho/coton. En effet, la quantité du carbone par rapport à la somme des taux de carbone dans la fraction argilo limoneuse des sols sont de $89,89 \%$ dans le système de culture coton/sorgho et de $82,89 \%$ dans le système de culture sorgho/coton. Les engrais appliqués à la culture du coton auraient favorisé la minéralisation rapide de la matière organique, d'où cette concentration du carbone dans la fraction fine des sols.

Les teneurs en carbone associées à la fraction $\mathrm{F}(200-2000 \mu \mathrm{m})$ et à la fraction F (50-200 $\mu \mathrm{m})$ sont plus élevées dans les sols des systèmes de culture arachide/sorgho et maïs/arachide. Ceci peut être attribué à l'arachide qui est un fixateur d'azote atmosphérique, ainsi que l'apport de la matière organique provenant des résidus du maïs et de l'arachide qui se minéralisent facilement.

Comme pour le carbone, les teneurs d'azote total sont concentrées dans la fraction argilo- limoneuse F $(0-50 \mu \mathrm{m})$. Christensen (2001) et Pallo et al. (2009) ont montré que le rapport $\mathrm{C} / \mathrm{N}$ de la matière organique associée aux différentes fractions baissent avec la taille de ces dernières. Cette diminution serait 
indicatrice d'une augmentation du degré d'humification des produits organiques [Guggenberger et al. (1994)] et d'un bon niveau de dispersion de l'échantillon. Elle serait aussi liée à une décroissance significative des phénols dérivés de la lignine des fractions grossières aux fractions fines Amelung et al. (1999). Les résultats obtenus au niveau du système de culture maïs/arachide concordent avec ces données. En effet, les rapports $\mathrm{C} / \mathrm{N}$ de la fraction $\mathrm{F}(0-50 \mu \mathrm{m})$ sont généralement plus faibles que ceux de la matière organique qui se trouvent dans les fractions sableuses. Ceci serait dû à la nature des matières organiques associées à cette fraction argilo-limoneuse: association de débris de parois végétales ou fongiques et de micro-agrégats organo-limoneux dans la fraction $(2-20 \mu \mathrm{m})$, dominance des matières organiques à caractère amorphe dans les fractions argileuses (Feller, 1995). Si une telle décroissance du rapport $\mathrm{C} / \mathrm{N}$ de la fraction $\mathrm{F}(200-2000 \mu \mathrm{m})$ à la fraction $\mathrm{F}(0-50 \mu \mathrm{m}) \mathrm{a}$ été obersvée dans les sols du système de culture maïs/arachide, c'est le phénomène inverse qui est observé dans ceux des systèmes de culture coton/sorgho, arachide/sorgho et sorgho/coton. Il serait dû aux très faibles teneurs en matière organique de ces sols et à leurs textures très grossières.

\section{Conclusion}

Les pratiques culturales paysannes se caractérisent par de faibles apports organominéraux conduisant à une baisse $\mathrm{du} \mathrm{pH}$ des sols de la plupart des systèmes de culture étudiés. En outre, ils ne compensent pas les exportations hors des parcelles des éléments nutritifs des sols car les teneurs en éléments minéraux ont aussi baissé. Les pratiques culturales ont également entraîné une baisse progressive de la matière organique des sols étudiés, rendant ainsi toutes les parcelles suivies sensibles à l'érosion. Les résultats obtenus suggèrent un certains nombre de recommandations pratiques pour améliorer la gestion de la fertilité des sols afin d'assurer durablement les productions agricoles :

- mettre un accent particulier sur la production et la diffusion des technologies de transformation des biomasses en matière fertilisante ;

- sensibiliser les producteurs à associer les fumures organiques aux engrais minéraux et à l'utilisation des légumineuses dans les systèmes de culture.

Dans le contexte actuel où les prix des engrais continuent d'augmenter alors que les prix des produits agricoles sont en baisse, la recherche doit s'orienter vers les systèmes de culture efficients et qui permettent de minimiser les coûts des engrais minéraux. Les investigations futures doivent viser davantage la mise au point des systèmes de culture intégrés et durables dans les savanes du Tchad

\section{REFERENCES}

Abdoulaye AA, Hofs JL, Mergeai G. 2006. Relever les défis environnementaux pour les filières cotonnières d'Afrique de l'Ouest et du Centre. Biotechnol. Agron. Soc. Environ., 10(4): 351-359.

Amelung W, Flach KW, Zech W. 1999. Lignin in particle size fractions of native grassland soils as influenced by climate. Soil Sci. Soc. Am. J., 63: 1222 - 1228.

Bado BV. 2002. Rôle des légumineuses sur la fertilité des sols ferrugineux tropicaux des zones guinéenne et soudanienne du Burkina Faso. Thèse $\mathrm{PhD}$, Faculté des Sciences de l'Agriculture et de l'Alimentation, Université de Laval, Quebec/Canada, 184 p.

Balesdent J, Chenu C, Balabane M. 2000. Relationship of soil organic matter dynamics to physical protection and tillage. Soil and Tillage Research, 35: 215-230.

Berger M. 1996. L'amélioration de la fumure organique en Afrique soudano sahélienne. Agriculture et Développement, numéro hors-série, CIRAD-CA, Montpellier/France, 58 p. 
Bremner JM. 1965. Total nitrogen. In Methods of Soil Analysis (part 2), Black CA (ed). American Society of Agronomy: Madison, WI, 9; 1149-1178.

Christensen BT. 2001. Physical fractionation of soil and structural functional complexity in organic matter turnover. European Journal of Soil Science, 52: 345-353.

Division de la Statistique Agricole. 2006. Données statistiques sur les productions agricoles du Tchad, $2 \mathrm{P}$.

Djondang K. 2003. Gestion d'exploitations agricoles dans un contexte de culture de coton: L'exemple de la zone soudanienne au Tchad. Thèse, Economie rurale, Institut National Polytechnique de Toulouse/France, p. 263.

Feller C. 1979. Une méthode de fractionnement de la matière organique des sols: application aux sols tropicaux, à texture grossières, très pauvre en humus. Cah. ORSTOM, série. Pédo., 17: 339346.

Feller C. 1995. La matière organique dans les sols tropicaux à argile. Recherche de compartiments organiques fonctionnels. Une approche granulométrique. Collection TDM n¹44, 5 microfiches, ORSTOM, Paris/France.

Ganry F, OIiver R, Hien E, Traaré K, Failler C, Gigou J, Thuries L. 2006. Gestion des apports de matière organique pour accroitre les stocks de carbone en région soudano-sahélienne. Les Matières Organiques en France. Etat de l'Art et Prospectives. CIRAD/IRD/INERA/IER, Carqueiranne/ France.

Guggenberger G, Christensen BT, Zech W. 1994. Land-use effects on the composition of organic matter in particlesize separates of soil. 1. Lignin and carbohydrate signature. European Journal of Soil Science, 45: 449-458.
Lompo F, Bonzi M, Bado BV, Gnankambary Z, Ouandaogo N, Sedogo MP, YaoKoamé A. 2007. Effets des modes de gestion de la fertilité des sols sur la solubilisation des phosphates naturels dans un Lixisol en zone nordsoudanienne du Burkina Faso. Sciences et Techniques, Série Sciences Naturelles et Agronomie, 29(1-2).

Nuttens F. 2001. La zone soudanienne du Tchad. Cédérom, ONDR/SCAC, Moundou / Tchad.

Pallo FJP, Sawadogo N, Zombré NP, Sedogo MP. 2009. Statut de la matière organique des sols de la zone nord soudanienne au Burkina Faso. Biotechnol. Agron. Soc. Environ., 13(1): 139-142.

Piéri C. 1989. Fertilité des Terres de Savanes. Bilan de Trente Ans de Recherche et de Développement au Sud du Sahara. Ministère Français de la Coopération et du Développement et CIRAD-IRAT: Paris, France; 444 p.

Richard L, Djoulet D. 1986. La fertilité des sols et son évolution. Zone cotonnière du Tchad. Coton Fibre Tropical, Sér. Documents, Etudes et Synthèse Supplément No. 6.

Sedogo PM. 1993. Evolution des sols ferrugineux lessivés sous culture : incidence des modes de gestion sur la fertilité. Thèse, Sciences naturelles (Agronomie-écologie-pédologie), Université d'Abidjan, Côte d'Ivoire, 330 p.

Walkley A, Black IA. 1934. An examination of the Degtjareff method for determining soil organic matter and a proposed modification of the chromic acid titration method. Soil Science, 37: 29-38. 